\title{
High-Energy Collision-Induced Dissociation of Multiply Charged Polypeptides Produced by Electrospray
}

\author{
Daniele Fabris, Michele Kelly, Constance Murphy, Zhuchun Wu, \\ and Catherine Fenselau \\ Department of Chemistry and Biochemistry, University of Maryland Baltimore County, \\ Structural Biochemistry Center, Baltimore, Maryland, USA
}

The recent commercial implementation of an electrospray source on a four-sector mass spectrometer has allowed the study of high-energy collisional activation of multiply charged cations. With this configuration, higher mass-to-charge ratios can be accommodated in both precursor ion selection and fragment ion detection. Good mass accuracy facilitates analysis of fragment ions and allows more reliable mechanistic correlation of these fragments. A convenient scheme was devised to permit the use of kilovolt potentials in both MS-I and MS-II, with precursors of varying charge states. Algorithms were devised to assign masses of different types of multiply charged fragment ions. Nine polypeptides were studied in the mass range 2000-5000 Da. Through this entire mass range, fragment ions were observed to be amply formed by cleavages in both the backbone and side chains, analogous to highenergy collisional activation of singly charged ions. This stands in sharp contrast to the patterns reported with low-energy, multiple collisions. Abundances of sequence ion series are influenced by the positions of basic residues. Analysis of charge distributions in fragment ions also indicates that the charges tend to be spread out across the peptides. (J Am Soc Mass Spectrom 1993, 4, 652-661)

$\mathrm{N}$ ew possibilities in the study of large biological polymers are offered by collisional activation of multiply charged ions generated by electrospray ionization (ESI). Multiple charging allows higher molecular weight precursors to be collisionally activated and enhances fragmentation in these heavier species.

To date, most reports of collisional activation of selected multiply charged peptides have involved triple quadrupole instruments using multiple low-energy collisions [1-4]. Peptide precursors with mixed charge states have been fragmented by low-energy collisions in the ESI source [4-7]. High-energy collisional activation has been examined with doubly charged ions produced by fast-atom bombardment (FAB) [8-10].

The recent commercial implementation of an ESI source on a four-sector instrument has allowed the present study of the features of high-energy collisional activation of multiply charged ions. The higher mass accuracy and higher resolution of the four-sector instrument provide more precise assignment of fragment ions. The larger mass range permits observation of precursors and fragment ions beyond the mass-to-

Address reprint requests to Daniele Fabris, Department of Chermistry and Biochemistry, University of Maryland Baltimore County, Structural Biochemistry Center, 5401 Wilkens Avenue, Baltimore, MD 21228. charge ratio range of most quadrupole instruments. Peptides are studied here with molecular weights that fall across the mass range of most proteolytic peptides.

\section{Experimental}

Collision-induced dissociation (CID) mass spectra were obtained on a JEOL (Tokyo, Japan) HX110/HX110 four-sector mass spectrometer $\left(\mathrm{E}_{1} \mathrm{~B}_{1} \mathrm{E}_{2} \mathrm{~B}_{2}\right)$, under control of a DA5000 data system and equipped with an Analytica of Branford (Branford, CT) ESI source.

Melittin (2846.5 Da) and insulin B chain oxidized $(3495.9 \mathrm{Da})$ were purchased from Sigma Chemical Co. (St Louis, MO). Renin substrate tetradecapeptide (1759.0 Da); human parathyroid hormone (1-34) (4117.8 Da), (1-44) (5063.0 Da), and (44-68) (2836.1 Da); human glucagon-like peptide I amide (4111.5 Da); human immunodeficiency virus gp120 antigenic peptide $(2720.3 \mathrm{Da})$; and human adrenocorticotropin (ACTH) (18-39) CLIP (2465.7 Da) were purchased from Bachem, Inc. (Torrance, CA).

Solutions of analytes were prepared by dissolving 1 $\mathrm{mg}$ of sample in $1 \mathrm{~mL}$ of water/acetonitrile/glacial acetic acid (49:49:2) and were introduced into the ESI source with a syringe infusion pump (model 22, Harvard Apparatus, South Natick, MA) at a flow rate of 2 $\mu \mathrm{L} / \mathrm{min}$. Dry nitrogen at a temperature of $160{ }^{\circ} \mathrm{C}$ was 
introduced into the capillary region at a flow rate of 30 $\mathrm{L} / \mathrm{min}$.

Collisional activations were accomplished on positive multiply charged ions in the third field-free region with a grounded or floated collision cell [11] using xenon as the collision gas at a pressure sufficient to attenuate the primary ion beam by $75 \%$.

The tandem mass spectrometer was operated with the collision cell grounded using different settings for different charge states of these peptides with molecular weights between 1700 and $5100 \mathrm{Da}$ :

\section{$2^{+}$Precursor ion: $5 \mathrm{kV}$ acceleration voltage in MS-I and $10 \mathrm{kV}$ in MS-II; \\ $3^{+}$Precursor ion: $3 \mathrm{kV}$ MS-I and $9 \mathrm{kV}$ MS-II; \\ $4^{+}$Precursor ion: $2 \mathrm{kV}$ MS-I and $8 \mathrm{kV}$ MS-II; \\ $5^{+}$Precursor ion: $2 \mathrm{kV}$ MS-I and $10 \mathrm{kV}$ MS-II.}

To carry out collisional activation with the collision cell floated, an equation was derived to calculate the correct settings to allow all of the possible fragments produced by CID to pass through MS-II and be detected. When a fragment ion $m_{f}$ of charge state $y$ is formed from a precursor ion $m_{\mathrm{p}}$ of charge state $z$ inside an electrically floated cell, its energy is

$$
u_{\mathrm{f}}=\left(m_{\mathrm{f}} / m_{\mathrm{p}}\right) z e\left(V_{\mathrm{a}}-V_{\mathrm{c}}\right)+y e V_{\mathrm{c}}
$$

where $V_{\mathrm{a}}$ is the accelerating voltage and $V_{\mathrm{c}}$ the voltage of the collision cell, or

$$
U_{\mathrm{f}}^{*}=\left(m_{\mathrm{f}}^{*} / m_{\mathrm{p}}\right) z e\left(V_{\mathrm{a}}-V_{\mathrm{c}}\right)+e \mathfrak{c} V_{\mathrm{c}}
$$

where $U_{f}^{*}=U_{\mathrm{f}} / y$, and $m_{\mathrm{f}}^{*}=m_{\mathrm{f}} / y$. Because electrostatic and magnetic sectors separate ions according to their kinetic energy to charge ratio $\left(U_{f}^{*}\right)$ and mass-tocharge ratio $\left(m_{\mathrm{f}}^{*}\right)$, respectively, the initial setting of MS-II for B/E linked scan should follow the equation $[10,12]$,

$$
z e\left(V_{\mathrm{a}}-V_{\mathrm{c}}\right)=V_{2}-V_{\mathrm{c}}
$$

where $V_{2}$ is the initial voltage of MS-II. In these experiments, $V_{2}$ is always set at $10 \mathrm{kV}$. Using this equation, the following values were calculated:

$$
\begin{aligned}
& 2^{+} \text {Precursor: } 6 \mathrm{kV} \text { MS-I, } 2 \mathrm{kV} \text { collision cell, } \\
& \text { and } 10 \mathrm{kV} \text { in MS-II; } \\
& 3^{+} \text {Precursor: } 5 \mathrm{kV} \text { MS-I, } 2.50 \mathrm{kV} \text { cell, } \\
& \text { and } 10 \mathrm{kV} \text { MS-II; } \\
& 4^{+} \text {Precursor: } 5 \mathrm{kV} \text { MS-I, } 3.33 \mathrm{kV} \text { cell, } \\
& \text { and } 10 \mathrm{kV} \text { MS-II; } \\
& 5^{+} \text {Precursor: } 4 \mathrm{kV} \text { MS-I, } 2.50 \mathrm{kV} \text { cell, }
\end{aligned}
$$

Low-energy CID experiments were performed for the melittin $4^{+}$precursor ion using the following settings:

$$
\begin{array}{ll}
4^{+} \text {Melittin: } & 7.0 \mathrm{kV} \mathrm{MS}-\mathrm{I}, 6.86 \mathrm{kV} \text { collision cell, } \\
& \text { and } 8 \mathrm{kV} \text { MS-II; } \\
4^{+} \text {Melittin: } & 7.9 \mathrm{kV} \mathrm{MS-I}, 7.89 \mathrm{kV} \text { collision cell, } \\
& \text { and } 8 \mathrm{kV} \text { MS-II. }
\end{array}
$$

The use of the floating cell is preferable because it allows high acceleration voltages in both MS-I and MS-II, leading to improved transmission and sensitivity. The spectra were obtained with $\mathrm{B} / \mathrm{E}$ linked scanning of MS- $\Pi$ from 0 to $\left([\mathrm{M}+\mathrm{H}]^{+}+10 \%\right)$ to collect the products with all of the possible charge states from $1^{+}$to the charge of the precursor ion. The scan rate for $m / z \quad 0-4500$ was $90 \mathrm{~s}$. The spectra presented are each the averaged profile of 12-18 scans recorded by the JEOL DA5000 data system. The resolution in MS-II was experimentally determined to be 1100 (50\% valley).

Multiply charged fragment ions were assigned using software written in our laboratory. This program starts with monoisotopic mass [13] values for the expected fragmentation of a singly charged precursor. From these values, the program calculates the massto-charge ratio values for all fragments with charge states up to that of the precursor ion, according to the algorithms proposed later in Results and Discussion, and matches the mass-to-charge ratio value of each real peak in the spectrum with those predicted. Monoisotopic masses for singly charged fragment ions were obtained using COMPOST software [14]. A more complete version of our software, allowing the calculation of the average mass values for the expected fragmentation, is now being developed in our laboratory.

\section{Results and Discussion}

Nine peptides, ranging in molecular weight from 1759.0 $\mathrm{Da}$ (renin substrate tetradecapeptide) to $5063.0 \mathrm{Da}$ [human parathyroid hormone (1-44)] were investigated. All of the charge states detected for molecular ions were used as precursors for the CID experiments in this study. Consistent with earlier studies [9, 10], transmission was found to be improved across the mass range when the collision cell was floated to provide kilovolt potentials across both MS-I and MS-II.

All of the high-energy CID spectra show similar general features (Figures 1-4): No matter which molecular weight or charge state is selected, precursor ions are detected as strong signals in the midrange of the mass-to-charge ratio scale, with fragment ions of various charge states recorded on both sides. The intensity and density of the fragment ion peaks decline, going to the extremes of the scan range.

A mass-analyzed ion kinetic energy spectrum of melittin $4^{+}$measured at the same experimental conditions as those in Figure 1 contained all of the same peaks, although broadened. This eliminates transmission problems in MS-II as a possible source of spectral 

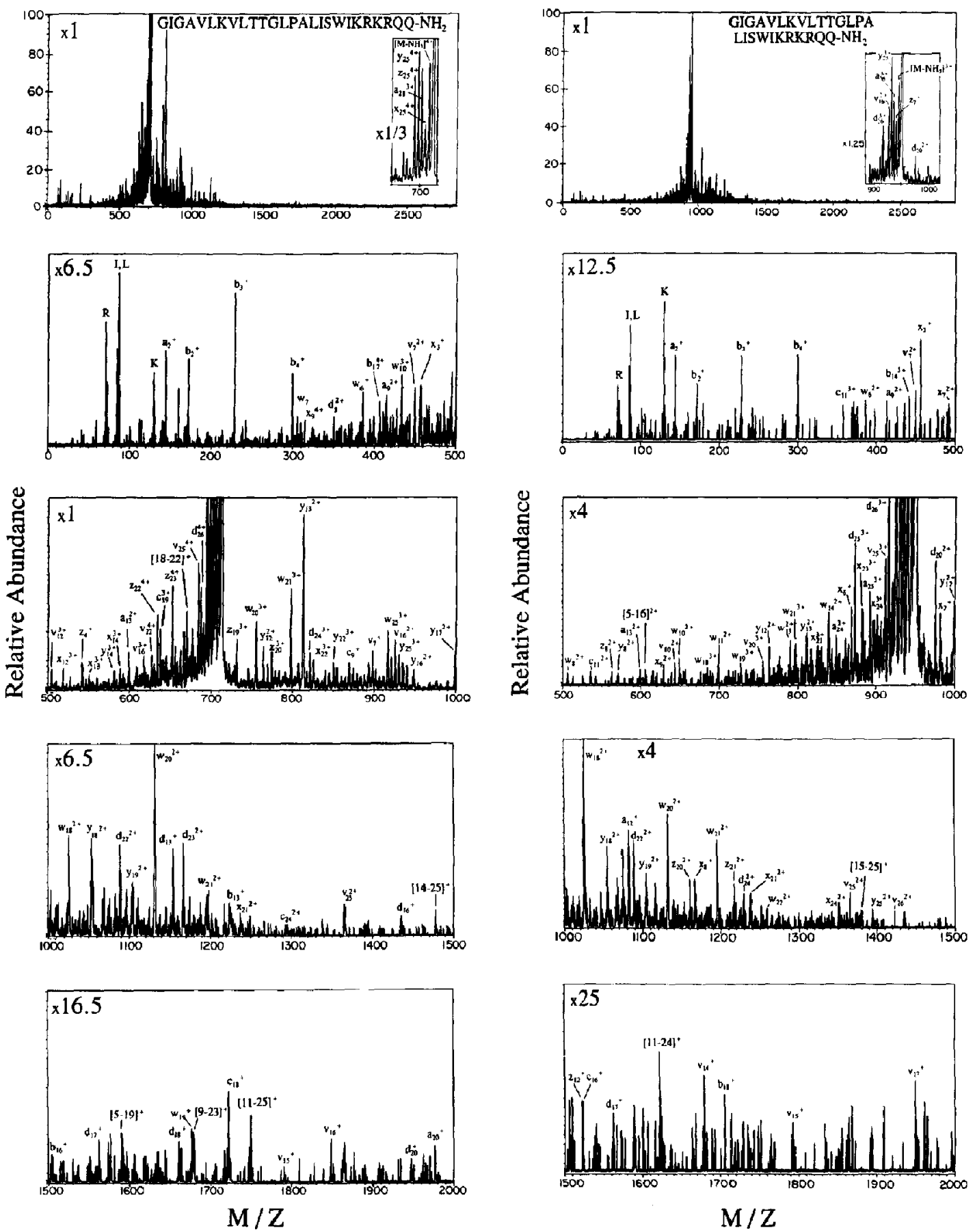

Figure 1. CID spectrum of melittin $4^{+}(2846.5$ Da): full-scan range spectrum $(m / z 0-2900)$ and enlargements.

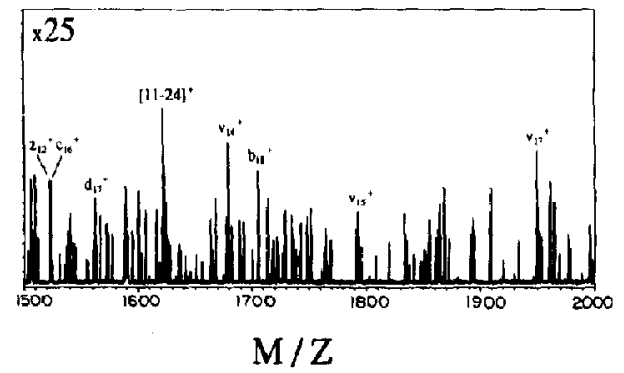

Figure 2. CID spectrum of melittin $3^{+}(2846.5 \mathrm{Da})$ : full-scan range spectrum $(\mathrm{m} / \mathrm{z} \quad 0-2900)$ and enlargements. 

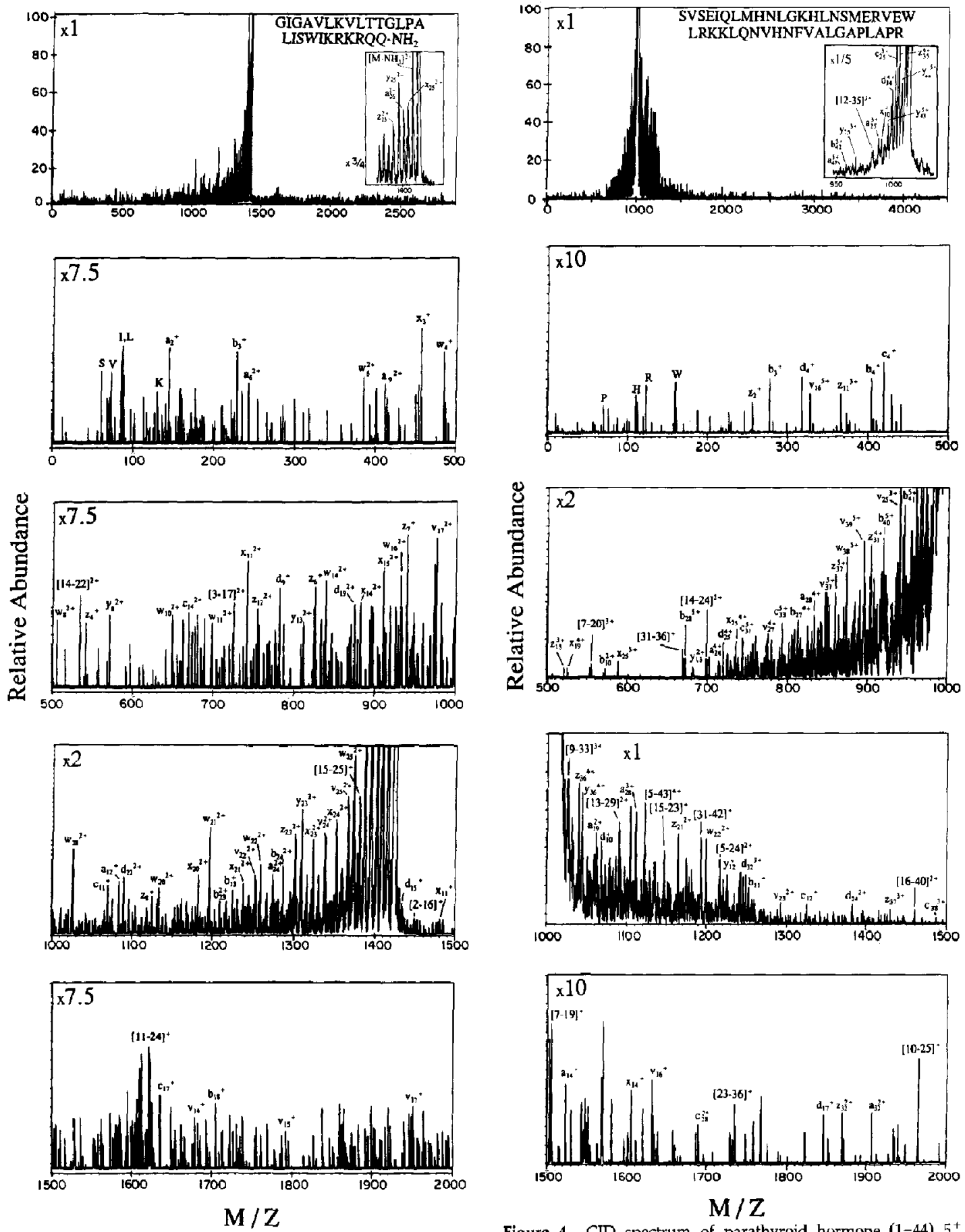

Figure 3. CID spectrum of melittin $2^{+}(2846.5 \mathrm{Da})$; full-scan range spectrum $(m / z \quad 0-2900)$ and enlargements.

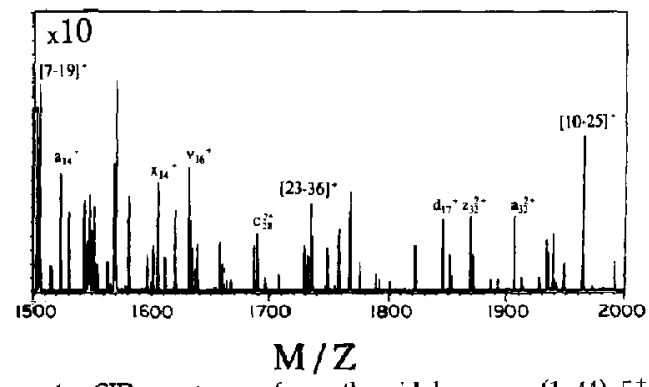

Figure 4. CID spectrum of parathyroid hormone (1-44) $5^{+}$ $(5063.0 \mathrm{Da})$ : full-scan range spectrum $(m / z \quad 0-4500)$ and enlargements of selected regions. 
distortion. Spectra of melittin $4^{+}$obtained with 570 and $57-\mathrm{eV}$ collision energy strongly resemble that of melittin $4^{+}$activated by multiple $565 \mathrm{eV}$ collisions in a triple quadrupole instrument [1]. These low-energy spectra contain low-intensity peaks in the low- and high-mass ranges [1]. The variety of fragment ions flanking the precursor is limited relative to the diversity observed in Figures 1-4.

The extent of fragmentation is maintained across the molecular weight range of peptides studied. Although spectra from different charge states of the same peptide differ quantitatively at the level of the individual peak (see, e.g., Figures 1-3 and Tables 1 and 2), the overall extent of fragmentation does not change dramatically at higher charge states.

It should be pointed out that the molecular weight of the peptide whose spectra are shown in Figures 4

and 5 is considerably heavier than that of precursor ions usually fragmented by collisional activation in four-sector instruments.

\section{Interpretation}

The interpretation of the spectra is based on the nomenclature presented by Biemann [15], which has been adapted to accommodate multiple charging. In the CID spectrum of a singly charged cation, $c_{-}, \mathrm{d}-, \mathrm{v}-$, $w_{-}$, and $y$-series ions originate by loss of neutral molecules, and the charge is still conferred by the proton added in the original ionization process. For a multiply charged fragment of one of these series, all of the charges are due to additional protons, and the mass of the fragment $\left(m_{\mathrm{f}}\right)$ can be calculated as shown:

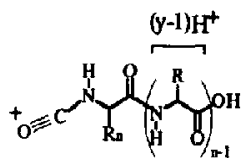

$\mathbf{X}_{\mathbf{n}}$

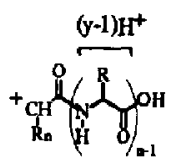

$\mathbf{Z n}_{\mathbf{n}}$

\section{for multiply protonated ions: $\quad \mathrm{m}_{\mathrm{f}}=\mathrm{m} / \mathrm{z} * \mathrm{y}-(\mathrm{y}-1)$}

where $m / z$ is the position of the peak in the spectrum and $y$ is the charge state of the fragment ion.

On the other hand, singly charged ions in the $a, b, x$, and $z$ series formed in high-energy CID processes do not retain an extra proton. In a multiply charged fragment of these series, all of the charges but one are carried by additional protons, and the mass of the fragment is calculated as shown:

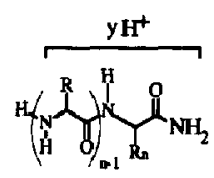

c.

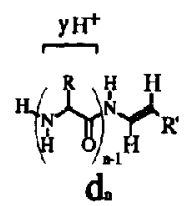

d.

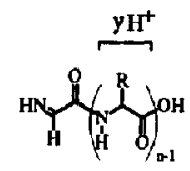

$V_{\text {n }}$

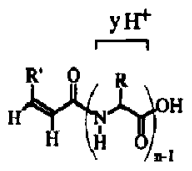

Wn

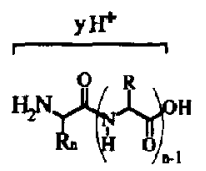

Yo

\section{for multiply protonated ions: $\quad \mathrm{mf}_{\mathrm{f}}=\mathrm{m} / \mathrm{z} * \mathrm{y}-\mathrm{y}$}

\section{Fragmentation Pattern}

The multiply charged precursor ions studied undergo fragmentation processes qualitatively similar to those observed in the spectra of products of high-energy collisional activation of singly charged ions. Both backbone and side-chain cleavages occur, immonium ions are formed, and some internal fragments are observed.

Figures 1-3 show the collisional activation spectra of melittin carrying four, three, and two protons, respectively. Interpretation is shown on the spectra, using the notation discussed above, and the presence and charge states of predicted sequence ions are summa- rized in Table 1 . The first column lists the amino acid sequence and the second column the residue number. The third column lists the fragments of a certain series originated by the $2^{+}$precursor ion, and so on. Different symbols are used to indicate the detection of different charge states of each fragment in each series. Table 1 confirms that fragmentation occurred across the entire peptide and that fragment ions are formed with all charge states up to that of the precursor.

Table 2, a summary of the sequence ions formed from ACTH (18-39) CLIP reveals another important consistency with the high-energy collisional activation of singly charged precursor ions. The peptide sequence of ACTH (18-39) CLIP carries all of its basic sites near 
Table 1. Summary of charge states observed for each sequence ion in the high-energy collisional activation spectrum of melittin $2^{+}, 3^{+}$, and $4^{+}$precursor ions ${ }^{a}$

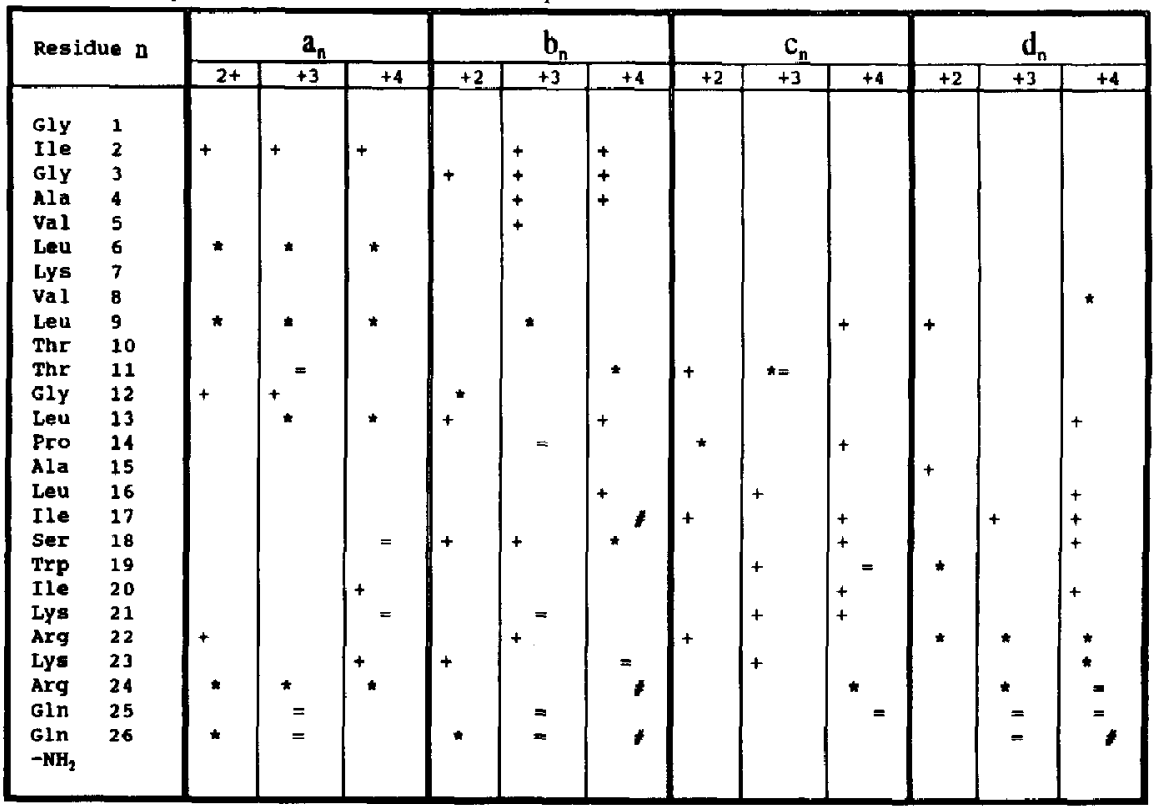

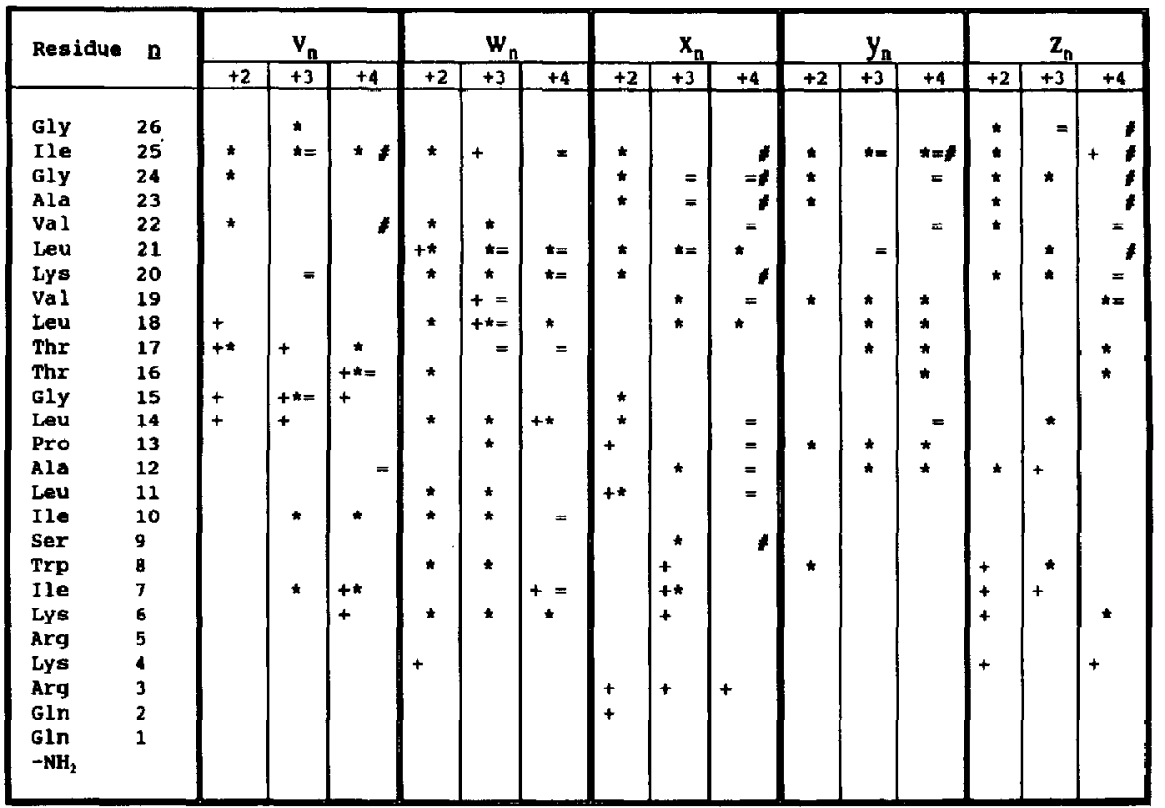

${ }^{a}$ The first column is the sequence of the peptide; the second $(r)$ is the numeration of the fragments according to the accepted nomenclature, begirning at the G-terminus for the series containing the C-terminus and at the N-terminus for the series containing the N-terminus; $(+) 1^{+} ;(*) 2^{+} ;(=) 3^{+} ;(\#)$ $4^{+}$.

the amino terminus: the free amino group, arginine-1, and lysine-4. Consistent with observations of singly charged precursors [16], a-d fragment ions that include the amino terminus are qualitatively and quantitatively favored.
Fragment ions in all charge states, from $1^{+}$to that of the precursor, are present in the same spectrum (e.g., Tables 1 and 2); however, the distribution is not uniform across the entire mass-to-charge ratio range. $A$ more complete sequence series can usually be assem- 
Table 2. Summary of charge states observed for each sequence ion in the high-energy collisional activation spectrum of ACTH (18-39) CLIP $3^{+}$and $2^{+}$precursor ions ${ }^{a}$

\begin{tabular}{|c|c|c|c|c|c|c|c|c|c|}
\hline \multirow[t]{2}{*}{ Residue } & \multirow[t]{2}{*}{ D } & \multicolumn{2}{|c|}{$a_{n}$} & \multicolumn{2}{|c|}{$b_{n}$} & \multicolumn{2}{|c|}{$\mathrm{c}_{\pi}$} & \multicolumn{2}{|c|}{$d_{n}$} \\
\hline & & +2 & +3 & +2 & +3 & +2 & +3 & +2 & +3 \\
\hline Arg & 1 & & & & & & & & \\
\hline Pro & 2 & + & & & & & + & & \\
\hline val & 3 & + & + & & & + & & + & \\
\hline Lys & 4 & & & + & & & & & \\
\hline val & 5 & + & * & + & & + & + & + & \\
\hline Tyr & 6 & + & $+*$ & + & + & & & & \\
\hline Pro & 7 & $*$ & $t=$ & & & & & & \\
\hline Asn & 8 & & * & & & & & & $=$ \\
\hline Gly & 9 & & & & & & & & \\
\hline Ala & 10 & & \pm & & & & & & \\
\hline Glu & 11 & & & + & & & & & \\
\hline AEp & 12 & + & * & $*$ & $=$ & + & & + & \\
\hline Glu & 13 & & & + & + & & & $\star$ & \\
\hline Ser & 14 & & $=$ & & & * & & + & \\
\hline Ala & 15 & $+*$ & & & * & & & & \\
\hline GIU & 16 & & * & + & * & & & * & \\
\hline Ala & 17 & * & & & * & & * & & \\
\hline Phe & 18 & * & * & + & & & & * & \\
\hline Pro & 19 & & * & & $=$ & & & & \\
\hline Leu & 20 & * & & & * & & * & * & \\
\hline Glu & 21 & & $=$ & & $*=$ & & * & $\star$ & \\
\hline Phe & 22 & & & & & & & & \\
\hline
\end{tabular}

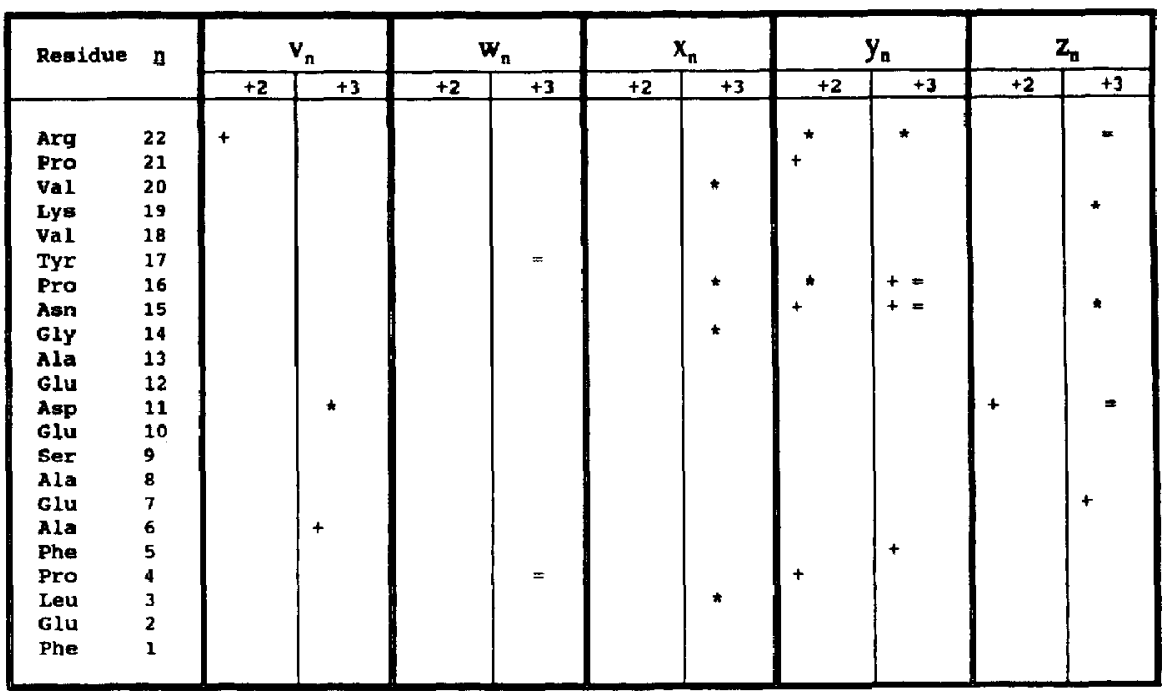

a The first column is the sequence of the peptide; the secand $(n)$ is the numeration of the fragments according to the accepted nomenclature; $(+) 1^{+} ;\left(^{*}\right) 2^{1} ;\left(=13^{+}\right.$.

bled by combining product ions with different numbers of charges, as well as the fragmentation proulucts of more than one precursor charge state.

Some differences are noted between the high-energy CID spectrum of melittin $2^{+}$produced by ESI and that produced by FAB [10]. These small differences may be due to different collision energies or different collision gases (Xe versus $H e$ ). The more interesting possibility that they are due to different internal energies in precursor ions produced by the different ionization processes is under investigation.

\section{Charge Sites}

Peaks with high mass-to-charge ratios (large fragments with low-charge states) are not detected with strong intersities in the spectra, suggestiny that larger fragments tend to have higher charge states. Similarly, few fragments are present with low-mass and high-charge states. The general patterns of the spectra (Figures 1-5) suggest that as a precursor loses residues, it also loses charges. In this way, the mass-to-charge ratios of the resulting fragments tend to remain close to a constant 

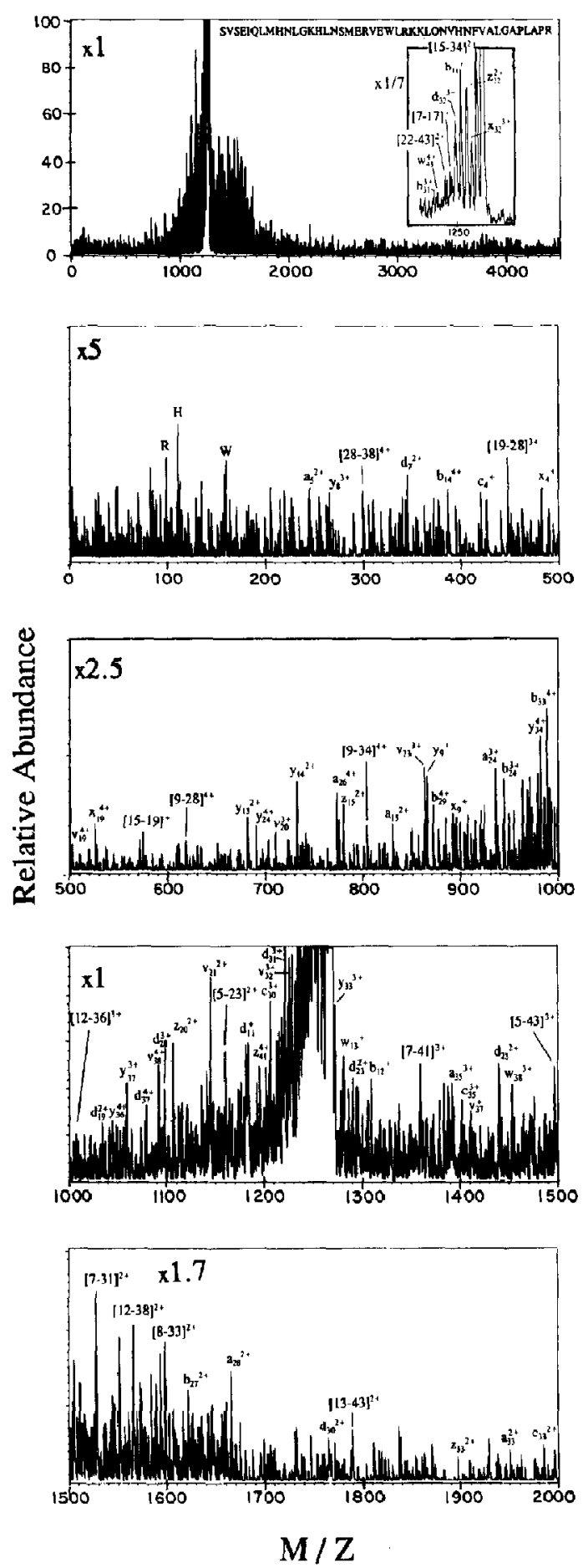

Figure 5. CID spectrum of parathyroid hormone (1-44) $4^{+}$ (5063.0 Da): full-scan range spectrum ( $m / \mathrm{z} 0-4500)$ and enlargements of selected regions.

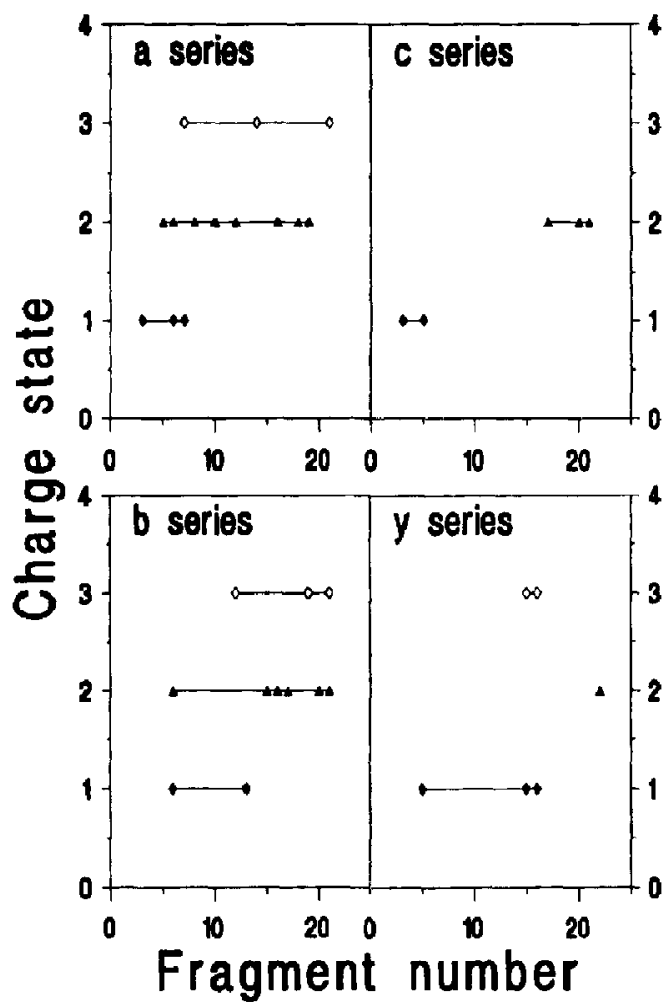

Figure 6. Summary of the number of amino acid residues in ions of different charge states in four sequence series formed from ACTH (18-39) CLIP $3^{+}$precursor ions by high-energy collisional activation: $(\bullet) 1^{+} ;(\bullet) 2^{+} ;\left(\right.$) $3^{+}$charge state.

number, and the peaks occur with striking frequency close to that of the precursor ion. Transmission problems in MS-II were excluded by energy analysis experiments (i.e., three-sector experiments) that showed spectra qualitatively similar to those obtained by the regular $B / E$ in four-sector experiments.

One feasible explanation is that charges tend to be distributed at rather even spatial intervals across the precursor ions. This is further examined in Figures 6 and 7 , in which charge state is plotted as a function of polymer length for ions in several of the sequence series formed in the activated decomposition of $3^{+}$ ACTH (18-39) CLIP and $5^{+}$human parathyroid hormone (1-44) precursors. In the peptide derived from $\mathrm{ACTH}$, all three of the basic sites for protonation occur in the first four residues in the polymer. It is possible for all three sites (amino terminus and arginine and lysine side chains) to be protonated in the $3^{+}$precursor ions; however, $a, b$, and $c$ ions carrying three charges are not detected until the chain length exceeds seven residues (Figure 6). Nlthough the $y$ ions are not abundant in the spectrum, they carry additional charges only as their chain length increases.

In Figure 7, charge states are plotted against chain 
length in two series of ions from each of the mechanistic sets discussed earlier (see Interpretation), formed from the $5^{+}$ions of human parathyroid hormone (1-44). Seven basic sites are distributed across this larger peptide, and not all are protonated in the $5^{+}$ precursor. Again, the number of protons or charges carried does not always coincide with the number of basic sites. There is a general correlation with chain length.

Complementary ions can provide artotler window on the location of charge sites. In the spectra of melittin, the fragments $b_{13}$ and $y_{13}$ originate by cleavage of the same proline peptide bond. The $b_{13}$ ion contains two basic sites, and the $y_{13}$ moiety contains four. The
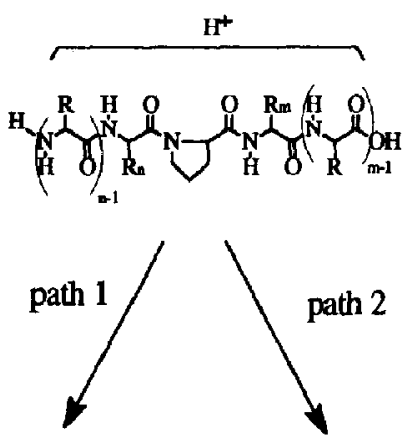

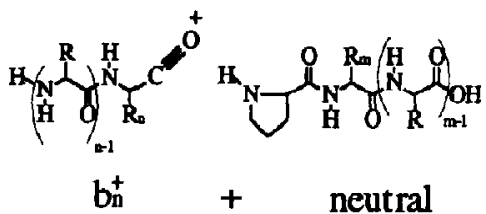

distribution of charges between the halves may reflect the distribution of charges in the precursor. In the CID spectrum of melittin $4^{+}$, the ratio of the intensity of $b_{13}^{+}$ to $y_{13}^{3+}$ is $18.5 \%$, and the ratio of $b_{13}^{2+}$ to $y_{13}^{2+}$ is $19.2 \%$, indicating that, regardless of charge locations, formation of $y_{13}$ ions is favored over formation of $b_{13}$ ions. The ratio of the sum of intensities of the $b_{13}^{+}$and $y_{13}^{3+}$ ions to the sum of the intensities of the $b_{13}^{21}$ and $y_{13}^{2}$ ions is $15.3 \%$. This indicates that the charges are not primarily clustered in the basic residues close to the carboxyl terminus of the molecule and that at least one proton is present with good probability in the amino terminus moiety. No signal is detected for $\mathrm{b}_{13}^{3+}$ or $\mathrm{y}_{13}^{+}$, indicating that the localization of more than two of the four charges on the amino terminus half of the peptide is unlikely. It appears that in the majority of $4^{+}$ions, two protons reside on each half of the peptide. (The alternative possibility of intramolecular proton transfers during fragmentation processes has not been excluded.)

\section{Comparison with Activation by Multiple Low- Energy Collisions}

A study of low-energy activation of melittin in various charge states was reported by Barinaga et al. [1], from which spectra and tables of fragment ions obtained from $3^{+}$and $4^{+}$precursors allow direct comparison with high-energy CID. Most ions are assigned as b- or $y$-series ions, with a few low-mass a and $c$ ions, in contrast to the diverse selection noted in Table 1 and in Figure 1 in this report. The earlier investigators sug-
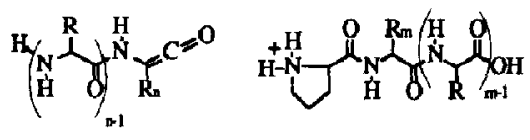

neutral

gest that complementary ion pairs are observed, but few cases could be found where total charge is conserved. The $b_{13}$ and $y_{13}$ ion pairs discussed above and others suggest a lower occurrence of charge or proton transfer in the present experiments, perhaps due to the use of xenon instead of nitrogen, to the different number of collisions, or to energy differences in the collisions.

The same group also published part of the spectrum of collisionally activated human parathyroid hormone (1-44) $5^{+}$ions [17]. This spectrum differs from ours (Figure 4) in both general and specific aspects. Fragment ions do not crowd in on both sides of the precursor mass-to-charge ratio value. Mostly $b$ - and y-series ions are assigned in the earlier work, whereas many internal fragments add to the diversity of this study.

\section{Conclusions}

Peptides with molecular weights above 5000 Da can be collisionally activated at kilovolt energies and analyzed through their entire mass ranges with a foursector mass spectrometer. The extensive fragmentation observed suggests that the implementation of ESI on four sector instruments extends the use of collisional activation through the mass range of most peptides produced by protease digestion of proteins. Transmission was sustained by floating the collision cell to provide kilovolt potentials across both MS-I and MS-II. Multiple charging also increased kinetic energy and transmission. 


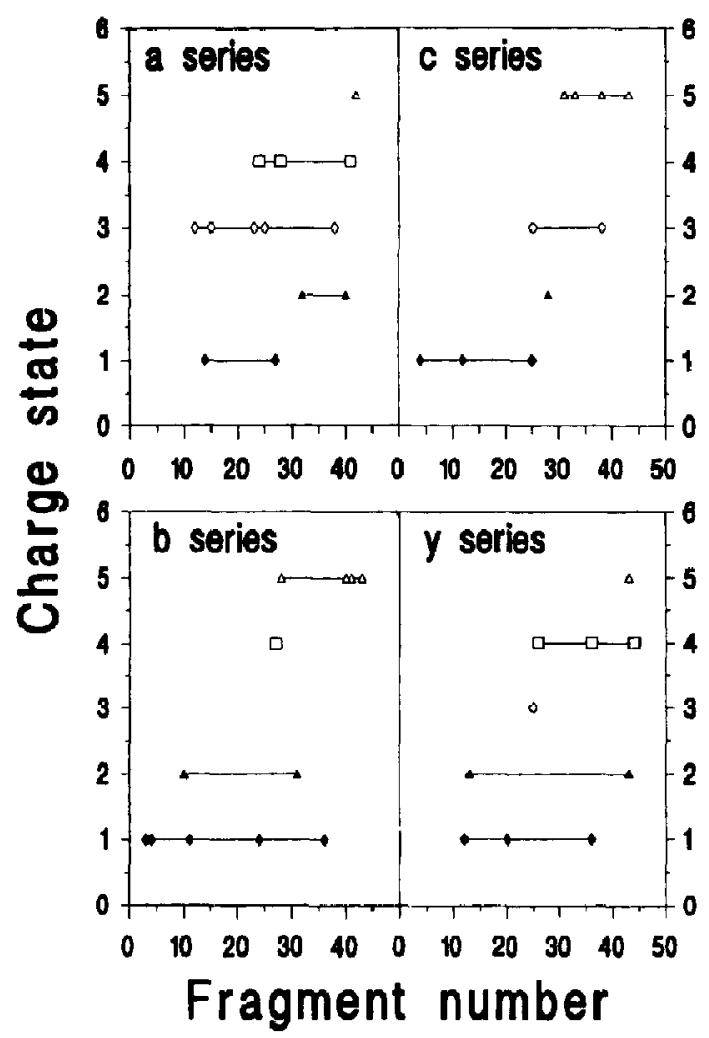

Figure 7. Summary of the number of amino acid residues in ions of different charge states in four sequence series formed from human parathyroid hormone $(1-44) 5^{+}$precursor ions by high-energy collisional activation: $(\bullet) 1^{+} ;(\Delta) 2^{+} ;(\diamond) 3^{+} ;(\square)$ $4^{+} ;(\Delta) 5^{+}$charge state.

The mass accuracy provided by the double-focusing analyzers allowed reliable assignment of many fragment ions, which were found to be formed by decomposition processes analogous to those familiar in singly charged peptides. Side-chain fragmentation and a much richer selection of ions were detected than has been observed with lower energy activation. The additional complexity of fragment ions with variable charge states strongly recommends computer-supported prediction of fragments and interpretation of spectra.

The spectra studied here indicate that the positions of strongly basic residues influence which fragment ions are detected, presumably reflecting charge localization analogous to that observed in fragmentation of singly charged peptides. At the same time, analysis of charge patterns in fragment ions suggests that charges are accommodated as far apart as possible on these relatively small peptides. This is particularly apparent in the species with more basic sites than charges.

\section{Acknowledgment}

This research was supported by grants from the National Science Foundation and from Sterling Winthrop, Inc.

\section{References}

1. Barinaga, C. J.; Edmonds, C. G.; Udseth, H. R.; Smith, R. D. Rapid Commun. Mass Spectrom. 1989, 3, 160-164.

2. Hunt, D. F.; Michel, H.; Dickinson, 'I. A.; Shabanowitz, J.; Cox, A. L.; Sakaguchi, K.; Appella, E.; Grey, H. M.; Sette, A. Science 1992, 256, 1817-1820.

3. Carr, S. A.; Hemling, M. E.; Bean, M. F.; Roberts, G. D. Anal. Chetr. 1991, 63, 2802-2824.

4. Smith, R. D.; Loo, J. A.; Ogorzalek Loo, R. R.; Busman, M.; Udseth, H. R. Mass Spectrom. Rev. 1991, 10, 359-451.

5. Aleksandrov, M. L.; Gall, L. N.; Krasnov, N. V.; Nikolaev, V. I; Paulenko, V. A.; Shkurou, V. A. Dokl. Akad. Nauk SSSR 1984, 277, 379.

6. Katta, V; Chowdhury, S. K.; Chait, B. T. Anal. Chem. 1991, 63, 174-178.

7. Meng, C. K.; McEwen, C. N.; Larsen, B. S. Rapid Commun. Mass Spectrom. 1990, 4, 151-155.

8. Barber, M.; Bell, D. J.; Morris, M.; Tetler, L. W.; Woods, M. D.; Monaghan, J. J.; Morden, W. E. Org. Mass Spectrom. 1989, $24,504-510$.

9. Orlando, R.; Fenselau, C. Org. Mass Spectrom. 1991, 26 , 664-666.

10. Orlando, R.; Boyd, R. K. Org. Mass Spectrom. 1992, 27. 151155.

11. Sato, K.; Asada, T.; Ishihara, M.; Kunihiro, F.; Kammei, Y.; Kubota, E.; Costello, C. E.; Martin, S. A.; Scoble, H. A.; Biemann, K. Anal. Chem. 1987, 59, 1652-1654.

12. Wu, Z.; Bordas-Nagy, J.; Fenselau, C. C. Org. Mass Spectrom. 1991, 26, 908-911.

13. Yerghey, J.; Heller, D.; Hansen, G.; Cotter, R. J.; Fenselau, C. Anal. Chem. 1983, 55, 353-356.

14. Papayannopoulos, I. A.; Biemann, K. I. Am. Soc. Mass Spectrom. 1991, 2, 174-177.

15. Biemann, K. Anntu. Rev. Biochem. 1992, 61, 977-1010.

16. Johnson, R. S,; Martin, S. A,; Biemann, K. Int. J. Mass Spectrom. Ion Proc. 1988, 86, 137-154.

17. Smith, R. D.; Loo, J. A.; Barinaga, C. J.; Edmonds, C. G.; Udseth, H. R. J. Am. Soc. Mass Spectrom. 1990, 1, 53-65. 